\title{
A Belief Rule based Expert System to Assess Clinical Bronchopneumonia Suspicion
}

\author{
Razuan Karim \\ Department of Computer Science \\ and Engineering \\ University of Science and \\ Technology Chittagong \\ Chittagong, Bangladesh \\ karim7@usa.com
}

\author{
Karl Andersson \\ Pervasive and Mobile Computing \\ Laboratory \\ Luleå University of Technology \\ Skellefteå, Sweden \\ karl.andersson@1tu.se
}

\author{
Mohammad Shahadat Hossain \\ Department of Computer Science \\ and Engineering \\ University of Chittagong \\ Chittagong, Bangladesh \\ hossain_ms@cu.ac.bd
}

\author{
Md. Jasim Uddin \\ Department of Computer Science and Engineering \\ University of Science and Technology Chittagong \\ Chittagong, Bangladesh \\ jasim_self@yahoo.com
}

\author{
Md. Perveg Meah \\ Department of Computer Science and Engineering \\ University of Science and Technology Chittagong \\ Chittagong, Bangladesh \\ jonysmile110@gmail.com
}

\begin{abstract}
Bronchopneumonia is an acute or chronic inflammation of the lungs, in which the alveoli and/or interstitial are affected. Usually the diagnosis of Bronchopneumonia is carried out using signs and symptoms of this disease, which cannot be measured since they consist of various types of uncertainty. Consequently, traditional disease diagnosis, which is performed by a physician, cannot deliver accurate results. Therefore, this paper presents the design, development and application of an expert system for assessing the suspicion of Bronchopneumonia under uncertainty. The Belief Rule-Based Inference Methodology using the Evidential Reasoning (RIMER) approach was adopted to develop this expert system, which is named the Belief Rule-Based Expert System (BRBES). The system can handle various types of uncertainty in knowledge representation and inference procedures. The knowledge base of this system was constructed by using real patient data and expert opinion. Practical case studies were used to validate the system. The system-generated results are more effective and reliable in terms of accuracy than from the results generated by a manual system.
\end{abstract}

Keywords-Belief Rule Base; Uncertainty; RIMER; Bronchopneumonia, Expert System; Inference

\section{INTRODUCTION}

Bronchopneumonia is the acute inflammation of the walls of the bronchioles. It is a type of pneumonia characterized by multiple foci of isolated, acute consolidation, affecting one or more pulmonary lobules [1]. They take the fifth place in the statistics of diseases causing death. So, diagnosis of Bronchopneumonia is an important issue. Bronchopneumonia is a type of pneumonia. There are two types of pneumonia: lobar and bronchial. Lobar pneumonia affects one or more sections, or lobes, or the lungs. Bronchopneumonia affects both lungs and the bronchi. Bronchopneumonia can be mild or severe, with viral bronchopneumonia usually being less severe [2].
Medical diagnosis is a process to detect a disease by measuring its specific signs and symptoms in a patient. Signs are observed by a physician and symptoms are expressed by the patient. Accuracy in measuring process is very important factor. However, the accuracy of measurement may become doubtful at any phase of diagnosis. A patient may not be able to precisely express their feelings and doctors may not be certain about the condition of signs. Sometimes, incomplete data are in hand which jeopardize to reach a decision about a disease in a patient. These uncertainties should be carefully overcome in order to gain an accurate diagnosis result.

In the case of Bronchopneumonia there are some significant signs and symptoms that are observed during the diagnosis process. The most common signs and symptoms of Bronchopneumonia are chest in drawing, chest pain, fever, respiratory distress rate, crepitation, cough with phlegm, chest pain, chills, headache, and fatigue. These signs and symptoms cannot be measured with certainty during the diagnosis procedure since there exist various types of uncertainty such as vagueness in data, imprecision in linguistic terms, incompleteness of data, ignorance and randomness. Consequently, traditional disease diagnosis cannot provide error-free results and thus one cannot reach to a straightforward algorithmic solution to diagnose Bronchopneumonia accurately. Therefore, an expert system is needed to handle this type of problem.

Until now the research in this domain has been carried out using traditional rule-based expert systems for Bronchopneumonia diagnosis [3]. However, the uncertainty issues were not fully overcome due to the dependency on normal if-then rules. The lack of strength to handle uncertainty leads to a notable weakness in those rule based expert systems.

This paper presents the development of an expert system (ES), based on a methodology, known as the belief rule-base (BRB) inference methodology using the evidential reasoning (RIMER) [4][5][6]. 
The remaining of the paper is structured as follows. Section two presents the related works. Section three provides an overview of RIMER methodology. Section four presents the architecture, design and implementation of the proposed BRBES. Experimental results and discussion are then presented. A conclusion is included to summarize the contribution of the research.

\section{RELATED WORK}

An expert system (ES) in the clinical domain can be defined as software that is designed to be a direct aid to clinical decision-making, in which the characteristics of an individual patient are matched to a computerized clinical knowledge base and patient-specific assessments or recommendations, is then presented to the clinician or the patient for a decision [7]. An expert system has two components: the knowledge base and the inference engine. The knowledge base can be constructed with proportional logic (PL), first-order logic (FOL) or fuzzy logic (FL) [8][9]. Reasoning mechanisms such as forward chaining and backward chaining are used to develop the inference engine [10]. PL and FOL are not equipped to capture uncertainty. However, FL can handle uncertainty due to vagueness and ambiguity. However, FL cannot handle other types of uncertainty such as ignorance and incompleteness that may exist in signs and symptoms of bronchopneumonia. Therefore, a knowledge base that can handle all types of uncertainty that exist with diagnosing bronchopneumonia must be developed. A relevant inference mechanism must also be adopted. Uncertain knowledge in diagnosing bronchopneumonia must be processed by using a refined knowledge base and an inference mechanism.

A recently developed Belief Rule-Based Inference Methodology Using the Evidential Reasoning (RIMER) approach [11][12][13] was used to design and develop the proposed decision support system. Uncertainty can be addressed by this methodology. This methodology consists of the Belief Rule Base (BRB) and the Evidential Reasoning (ER) algorithm. In RIMER, a rule base is designed with belief degrees embedded in all possible consequents of a rule. Inference in such a rule base is implemented using the evidential reasoning approach that can handle different types and degrees of uncertainty in signs and symptoms.

\section{OVERVIEW OF RIMER METHODOLOGY}

The RIMER approach consists of two basic components [10]. They are BRB to act as the knowledge base and ER to act as an inference engine.

\section{A. Abbreviations and Acronyms}

Belief rules are the key elements of a BRB, which include belief degree. It is an extended form of traditional If-Then rules. A belief rule consists of an antecedent part and a consequent part. The antecedent attribute takes referential values, and each consequent is associated with belief degrees [14].

The knowledge representation parameters are rule weights, antecedent attribute weights and belief degrees in consequents, which can handle uncertainty. A belief rule can be defined as follows:
$\mathrm{R}_{\mathrm{k}}$ : $\operatorname{if}\left(\mathrm{S}_{\mathrm{k} 1 \text { is }} \mathrm{A}_{1}^{\mathrm{k}}\right)$ and $\left(\mathrm{S}_{\mathrm{k} 2}\right.$ is $\left.\mathrm{A}_{2}^{\mathrm{k}}\right)$ and .... $\left(\mathrm{S}_{\mathrm{kTk}}\right.$ is $\left.\mathrm{A}_{1}^{\mathrm{kTk}}\right)$ then $\left\{\left(D_{1}\right.\right.$ is $\left.\beta_{1 \mathrm{k}}\right),\left(D_{2}\right.$ is $\left.\beta_{2 \mathrm{k}}\right), \ldots\left(\mathrm{D}_{\mathrm{N}}\right.$ is $\left.\left.\beta_{\mathrm{Nk}}\right)\right\}$

with a rule weight $\theta_{k}$ and attribute weights $\delta_{k 1}, \delta_{k 2}, \ldots \delta_{k T k}$ $\mathrm{k} \in\{1, \ldots, \mathrm{L}\}$

Here $S_{k 1}, S_{k 2}, \ldots, S_{\mathrm{k} T k}$ stand for the antecedent attributes in $k^{\text {th }}$ rule and each $A_{j}^{\mathrm{k}}\left(j \in\left\{1, \ldots, T_{k}\right\}\right)$ is one of the referential value of $\mathrm{S}_{k i} . T_{k}$ is the total number of antecedent attributes in the $k^{\text {th }}$ rule. $\beta(\mathrm{ik})$ is the belief degree to which $D(\mathrm{i})$ is believed to be the consequent if in the $k^{\text {th }}$ rule packet rule the antecedent part is satisfied. $\mathrm{L}$ is the total number of packet rules and $N$ is the number of all possible consequents in the belief rule base.

The following is an example of a belief rule where the distribution associated with the bronchopneumonia diagnosis:

$R_{k}:$ IF (Chest indrawing is Yes) AND (Chest pain is Severe) AND (Fever is High Grade) AND (Respiratory Distress Rate is Out of Range) AND (Crepitation is Yes) AND (Cough with phlegm is yes) THEN Bronchopneumonia is (High, 1.00), (Medium, 0.0) and (Low, 0.0)\} Here, \{(High, 1.00), (Medium, 0.0), (Low, 0.00)\}

This belief distribution states that, it is $100 \%$ sure that the condition of the bronchopneumonia is 'high'. A BRB given in the form shown in (1) represents functional mappings between antecedents and consequents with uncertainty. It is considered as a more realistic and informative scheme for uncertain knowledge representation. The BRB is established by using available up to date information regarding the inputs and outputs. Experts can directly assign the belief degrees. Then, the knowledge in the BRB can be used to execute inference for given inputs.

\section{B. Inference Procedure in $B R B$}

In RIMES methodology evidential reasoning or ER approach has been chosen to perform inference through the $\mathrm{BRB}$. According to RIMER approach the inference process for some inputs is accomplished through several tasks. The tasks includes performing input transformation, calculating activation weight of the rules, updating belief degrees and finally aggregating the rules to get the final result using ER algorithm. All these steps are described below.

\section{Input Transformation}

Before starting the inference process, the relationship between an input and each referential value in the antecedent of a rule needs to be determined. This part is known as input transformation. Input transformation is done so that an activation weight for each rule can be generated, that is no rule will be remained unobserved. A matching degree to which an input belongs to a referential value is calculated. It seems to transforming an input into a distribution on referential values using belief degrees. Input transform determines the matching degrees between an input and the referential values of all antecedents in a rule. The next step is to generate an activation weight for the rule. Activation weight of a rule is a measurement of the degree to which the antecedent part of the rule is activated by the input. Given the input for a BRB the activation weight $w_{k}$ for each $k^{\text {th }}$ rule can be calculated as follows. Consider an input after input-transformation corresponding to the $\mathrm{k}^{\text {th }}$ rule defined as in (1): 
$\left(\mathrm{S}_{\mathrm{k} 1}\right.$ is $\left.\mathrm{A}_{\mathrm{k} 1}, \alpha_{\mathrm{k} 1}\right) \wedge\left(\mathrm{S}_{\mathrm{k} 2}\right.$ is $\left.\mathrm{A}_{\mathrm{k} 2}, \alpha_{\mathrm{k} 2}\right) \wedge \ldots \wedge\left(\mathrm{S}_{\mathrm{kTk}}\right.$ is $\left.\mathrm{A}_{\mathrm{kTk}}, \alpha_{\mathrm{kTk}}\right)$

Where $\alpha_{k i}$ is the matching degree between input value for antecedent attribute $S_{\mathrm{ki}}$ and referential value, $\mathrm{A}_{\mathrm{ki}}$ in the $\mathrm{k}_{\mathrm{th}}$ rule $\left(i=1, \ldots, \mathrm{T}_{k}\right)$.

The total degree $\alpha \mathrm{k}$, to which the input matches the whole antecedent part of rule, can be calculated by using the following formula:

$$
\alpha_{k}=\operatorname{aggr}\left(\left(\delta_{k 1}, \alpha_{1}^{k}\right), \ldots,\left(\delta_{k T k}, \alpha_{1}^{T k}\right)\right)
$$

Where aggr is an aggregation function which should be selected carefully. In the case of a rule defined as in (3), the following simple weighted multiplicative aggregation function can be used:

Where

$$
\alpha_{k}=\prod_{i=1}^{T k}\left(\alpha_{i}^{k}\right)^{\delta_{k i}}
$$

$$
\bar{\delta}_{k i}=\frac{\delta_{k i}}{\max _{i=1 \ldots T k}\left(\delta_{k i}\right)}
$$

The above shown aggregation function is used in bronchopneumonia diagnosis to calculate the total matching degree $\alpha \mathrm{k}$. Next, the activation weight wk for rule can be generated by the following equation:

$$
\omega_{k}=\frac{\theta_{\mathrm{k}} \cdot \alpha_{\mathrm{k}}}{\sum_{\mathrm{i}=1}^{\mathrm{L}} \theta_{\mathrm{i} \cdot \alpha_{\mathrm{i}}}}
$$

This activation weight will be zero if the $k^{\text {th }}$ rule is not activated.

If the sum of belief degrees associated with the consequents is less than zero for a rule then the rule is incomplete. The incompleteness of the consequent of a rule can also occur by its antecedents because of the lack of data. Such incompleteness should be taken into account in the inference process. Therefore, the existing belief degree is updated using the actual input information.

$$
\beta_{i k}=\bar{\beta}_{i k} \frac{\sum_{t=1}^{T_{k}}\left(\tau(t, k) \sum_{j=1}^{J_{i}} \alpha_{t j}\right)}{\sum_{t=1}^{T k} \tau(t, k)}
$$

Where $\beta_{i k}=$ Update Belief

$$
\tau(\mathrm{t}, \mathrm{k})=\left\{\begin{array}{c}
1, \text { if } \mu_{t} \text { is used to defining } R_{k}\left(t=1, \ldots \ldots, T_{k}\right) \\
0, \text { otherwise }
\end{array}\right.
$$

Rule aggregation measured by ER method, first, should model the belief rule-base signal determination in the ER framework taking each activated rules packet antecedent as a basic attribute. In this model, each rules activation weights acts like a basic attribute weight and each possible consequent of the BRB acts like each individual evaluation grade set for the basic attributes. A basic probability mass representation.

$$
\begin{gathered}
m_{j, k}=\omega_{k} \beta_{j, k}, j=1, \ldots, N \\
m_{D, k}=1-\sum_{J=1}^{N} m_{j, k} \\
m_{D, k}=1-\omega_{k} \sum_{J=1}^{N} \beta_{j, k} \\
\left.m_{D, I(k)} m_{j, k+1}\right] \\
\{D\}: m_{D, I(k+1)}=k_{I(k+1)}\left[m_{D, I(k)} m_{D, k+1}\right] \\
k_{I(k+1)}=\left[1-m_{j, I(k)} m_{j, k+1}+m_{j, I(k)} \times m_{D, K+1}+\right. \\
\left.\sum_{j=1, t=1}^{N} m_{j, I(k)} m_{t, k+1}\right]^{-1}
\end{gathered}
$$

FTC 2016 - Future Technologies Conference 2016 6-7 December 2016 | San Francisco, United States

$$
\left\{D_{j}\right\}: \beta_{j}=\frac{m_{j, I(l)}}{1-m_{d, I(L)}}
$$

To convert aggregated fuzzy values into numeric values, the following equation will be applied:

$$
y_{m}=\sum_{n=1}^{N} D_{n} * \beta_{n}(m)
$$

Here, $\beta_{n}(m)$ is utility value. In this case, utility values are $1.00,0.5$ and 0 . Using the equation the result can be converted into numerical.

\section{BRBES FOR ASSESSING BRONCHOPNEUMONIA SUSPICION}

This section presents the design, implementation, knowledge base construction and interface of the BRBES for diagnosing Bronchopneumonia.

\section{A. Archiecteure, Design, and Implementation of the BRBES}

The design of the system consists of data structure and program components that are essential to build a computer based system. It also considers the system organization pattern, which is known as architectural style. The architecture of the BRBES consists of user interface, a knowledge engineer, knowledge base, inference engine, documentation and knowledge refinement as shown in Figure 1. User interface interacts to a system user to get input data and to receive system generated output. Visual Basic 6.0 has been employed to develop the system interface. Knowledge engineer accommodates data from domain knowledge and expert constructs knowledge base by using belief rule base. MS SQL Server is a relational database used at the back-end to store and manipulate initial BRB, which is flexible and user friendly. The inference engine carries the tasks of input transformation, rule activation, rule update and rule aggregation by using ER.

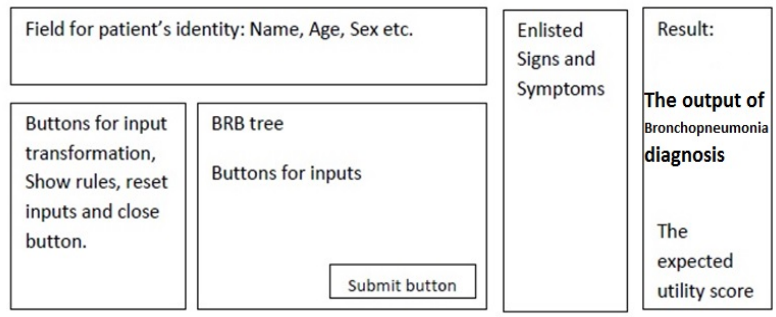

Fig. 1. The BRBES System Architecture

\section{B. Knowledge Base Contruction in BRB}

To construct the knowledge base for our BRB system prototype, a BRB framework was used following the British Guideline on the Management of Bronchopneumonia. In the framework, the input factor that determine suspicion are $\mathrm{A} 1=$ Chest Indrawing, A $2=$ Chest pain, A $3=$ Fever, A4=Respiratory Distress Rate, A5 $=$ Crepitation, A6=Cough with phlegm, and A7=Bronchopneumonia. This BRB consists of only the Bronchopneumonia rule base and is depicted in Figure 2. The rule base has six antecedent attributes. The total number of rules, $L$, is usually determined with the following method:

$$
\mathrm{L}=\prod_{i=1}^{t} J i
$$

Where $J_{1}=2, J_{2}=3, J_{3}=3, J_{4}=3, J_{5}=3, J_{6}=2$ so $L=$ $(2 * 3 * 3 * 3 * 3 * 2)=324$. Thus, the entire BRB consists of 324 
belief rules as illustrated in Table 2. It is assumed that all belief rules have equal rule weight and all antecedent attributes have equal weight. The initial belief base for the Bronchopneumonia suspicion BRB system is listed in Table 1.

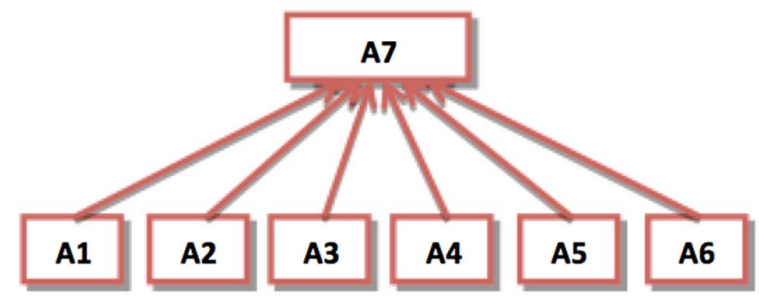

Fig. 2. BRB Framework for Bronchopneumonia Suspicion

An example belief rule of this BRB system as follows:

R1: IF (Chest indrawing is Yes) AND (Chest pain is Severe) AND (Fever is High Grade) AND (Respiratory Distress Rate is Out of Range) AND (Crepitation is Yes) AND (Cough with phlegm is yes) THEN Bronchopneumonia is $\{S(1.00), M(0.00), L(0.00)$ and $N(0.00)\}$

Here: $S=$ Severe, $M=$ Medium, $L=$ Low and $N=N i l$

In this belief rule, the belief degrees are attached to the six referential values of the consequent attribute. For example, having 10 patients whose clinical information such as "Chest indrawing is Yes", "Chest pain is Severe", "Fever is High Grade", "Respiratory Distress Rate is Out of Range", "Crepitation is Yes" and "Cough with phlegm is yes". This means that if the clinical information of the antecedent parts of the rule is evaluated with a high referential value of the corresponding antecedent attribute of the rule, then all patients are judged as severe regarding a diagnosis of Bronchopneumonia. Consequently, the rule can assign initial belief degrees to the consequents in the rule such as " 1 " to " $\mathrm{S}$ ", " 0 " to "M", " 0 " to "L" as shown in rule R1.

\section{BRBES Interface}

A system interface can be defined as the medium that enables interaction between the users and the system. Figure 3 illustrates a simple interface of the BRBES.

Here, the input antecedent for chest indrawing is yes, chest pain is severe, fever is high, respiratory distress rates is out of range and crepitation is high and cough with phlegm is no. The
FTC 2016 - Future Technologies Conference 2016 6-7 December 2016 | San Francisco, United States

BRBES generates the fuzzy value of the referential value. Then the system converts the fuzzy value into one numerical value by multiplying three utility factors. The three reference values are 1.0 for the High referential value, 0.5 for the Medium referential value and 0 for the Low referential value.

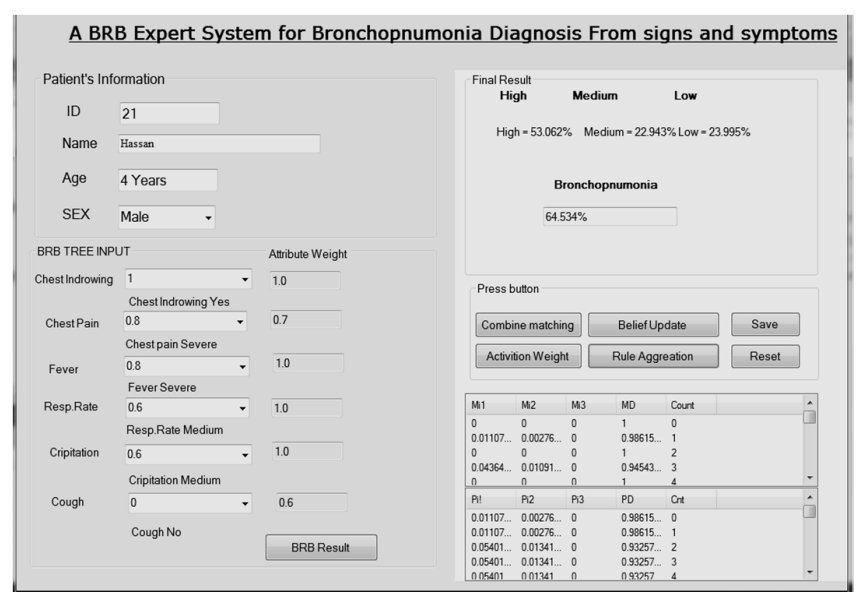

Fig. 3. BRBES Interface

The fuzzy output of the system is:

Bronchopneumonia (A7): \{(High, 53.062\%), (Medium, $22.943 \%$ ), (Low, 23.995\%)\}.

High referential value $=(53.062 \% * 1.00)=53.062 \%$, Medium referential value $=(22.943 \% * 0.5)=11.471 \%$, and Low referential value $=(23.995 \% * 0)=0 \%$.

Thus, the total system output is $(53.062 \%+11.471 \%+0 \%)=$ $64.534 \%$. The result of the system's diagnosis is $64.534 \%$ for the bronchopneumonia suspicion. This result is shown in Figure 3.

\section{RESUlTS AND DisCUSSION}

In this research, leaf nodes data of the BRB were collected from patients who suffer from bronchopneumonia. Then the patient data were used in the BRBES to assess broncho-pneumonia suspicion. Expert opinion on the broncho-pneumonia suspicion was also collected as shown in Table 2. If a patient has bronchopneumonia, then the benchmark datum is 1 ; otherwise, it is 0 . The data set consists of fifty samples. For simplicity, data for only ten patients is presented in Table 2. 
TABLE I. INITIAL BELIEF RULE BASE

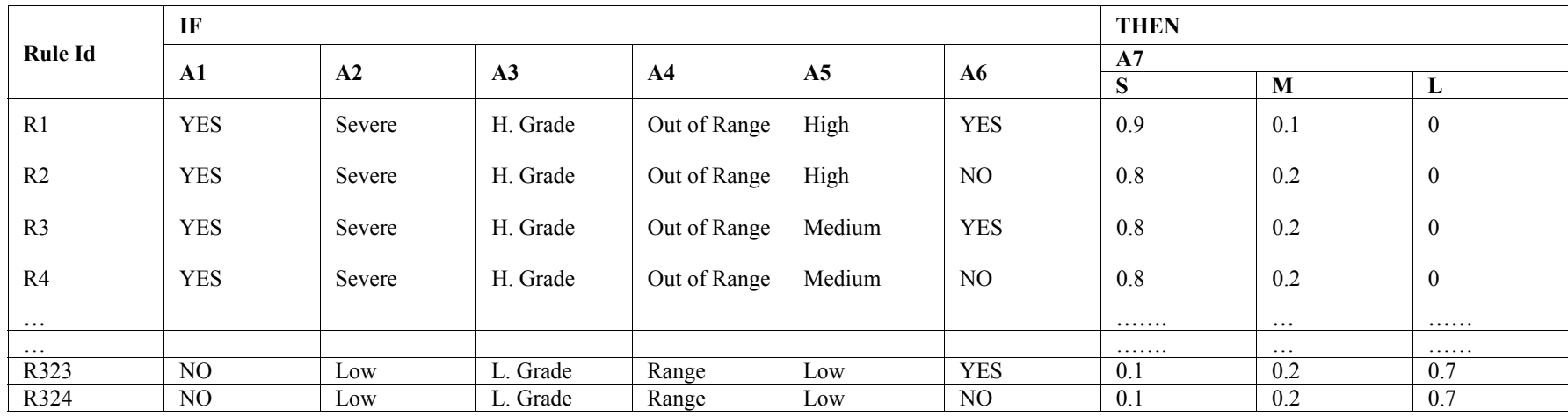

TABLE II. BRONCHOPNEUMONIA SUSPICION BY BRBES AND EXPERT

\begin{tabular}{|c|c|c|c|c|c|c|c|c|c|}
\hline Patient No & A1 & $\mathbf{A 2}$ & $\mathbf{A 3}$ & A4 & A5 & A6 & $\begin{array}{l}\text { BRB } \\
\text { Expert } \\
\text { System }\end{array}$ & $\begin{array}{l}\text { Expert } \\
\text { Opinion }\end{array}$ & Bench-mark \\
\hline 1 & YES & Severe & H. Grade & Out of Range & High & YES & 95 & 90 & 1 \\
\hline 2 & YES & Severe & H. Grade & Range & Low & YES & 64.55 & 60 & 1 \\
\hline 3 & YES & Severe & M. Grade & Range & Medium & YES & 58.79 & 50 & 1 \\
\hline 4 & YES & Low & M. Grade & Medium & High & NO & 48.74 & 45 & 1 \\
\hline 5 & YES & Low & L. Grade & Medium & High & YES & 56 & 50 & 1 \\
\hline 6 & NO & Severe & H. Grade & Out of Range & Low & YES & 19.82 & 18 & 1 \\
\hline 8 & YES & Moderate & L. Grade & Medium & High & NO & 63.06 & 60 & 1 \\
\hline 9 & YES & Severe & L. Grade & Range & Medium & NO & 58.14 & 55 & 1 \\
\hline 10 & YES & Severe & M. Grade & Medium & Low & $\mathrm{NO}$ & 67.45 & 60 & 1 \\
\hline
\end{tabular}

The Receiver Operating Characteristic (ROC) curves can help effectively analyze the performance of the suspicion/ diagnosis tests that have ordinal or continuous results [15].

It can be used to test the results of the BRBES and the manual system/expert opinion results by using the benchmark results. The system performance can be measured by calculating the area under the curve (AUC) [15][16][17][18]

If the AUC of the BRBES is larger than the expert opinion, then the BRBES produces more accurate and reliable results. Figure 4 shows the two ROC curves. One represents the suspicion performances of the BRB system prototype, and the other is the result of the manual system/expert opinion. The ROC curve with a red line in Figure 4 illustrates the BRB system bronchopneumonia diagnosis while the curve with green line illustrates the manual system bronchopneumonia diagnosis. The AUC for the BRB system prototype is 0.952 $(95 \%$ confidence interval $=0.960-1.012)$, and the AUC for the expert opinion is 0.857 (95\% confidence interval $=0.939$ 1.014). From the AUC of the BRBES and expert opinion, the AUC for the BRBES is greater than the AUC for the expert opinion. This implies the results generated by the BRBES are better than the results generated by expert opinion. SPSS 16.0 was used to construct the ROC curve and to calculate the AUC of the curves.

The achievement of our research is to overcome the uncertainty problem involved in diagnosing bronchopneumonia, which cannot be overcome with the traditional rule-based system. The BRBES can handle various types of uncertainty such as ambiguity, vagueness, imprecision, ignorance, etc.

\section{CONCLUSION}

In this paper, we demonstrated the development and application of a BRBES to diagnose bronchopneumonia based on signs and symptoms. This BRBES used a methodology known as RIMER that handles various types of uncertainty

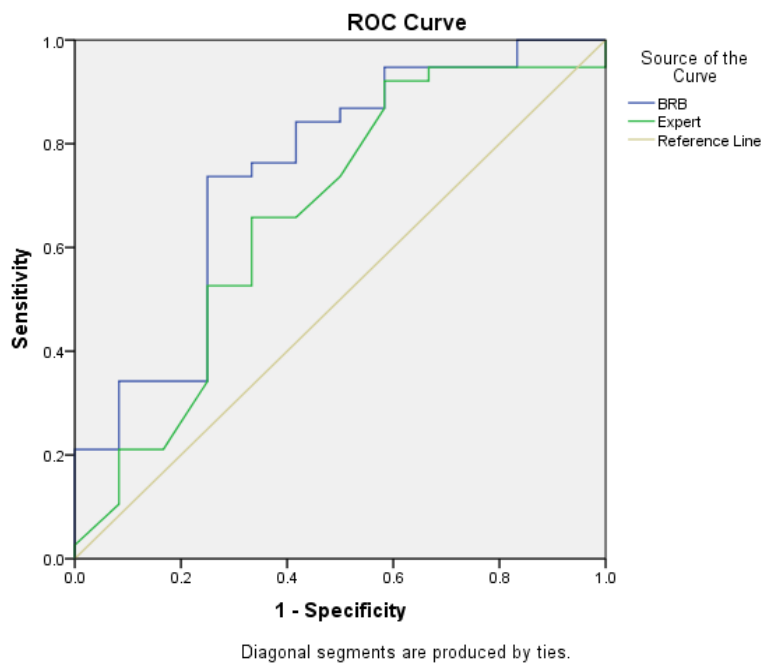

Fig. 4. ROC curves of bronchopneumonia suspicion between the BRBES and expert opinion 
found in domain knowledge. The BRBES is a robust tool that can aid in assessing bronchopneumonia suspicion. The system will help patients assess improvement in bronchopneumonia severity as well. This BRBES provides a percentage of the assessment, which is more reliable and informative than from a traditional expert's opinion that given without a degree of belief that is weighted with percentage value. Results generated by the BRBES were more reliable than the traditional expert opinion.

The system has strong potential in developing countries in Africa and Asia, in addition to other countries, where there is a lack of healthcare resources, diagnosis machinery and expert physicians.

\section{ACKNOWLEDGMENT}

We would like to extend our gratitude to the Swedish Research Council (under grant 2014-4251) for the funding and the provision of resources to conduct this research.

\section{REFERENCES}

[1] [Online]. Available: https://en.wikipedia.org/wiki/Bronchopneumon

[2] [Online].Available:

$\mathrm{http}: / / \mathrm{www} \cdot$ healthline.com/health/bronchopneumonia\#Overview1

[3] R. Smith. Knowledge-Based Systems Concepts, Techniques, Examples, 8 May 1985.

[4] S. Rahaman and M. S. Hossain. A belief rule based clinical decision support system to assess suspicion of heart failure from signs, symptoms and risk factors, Dhaka: IEEE; 2013, p. 1-6.

[5] H. Skalska and V. Freylich. Web-bootstrap estimate of area under ROC curve. Aust. J. Stat. 2006;35:325-30.

[6] J. A. Hanley. The Robustness of the "Binormal" Assumptions Used in Fitting ROC Curves. Med. Decis. Making 1988;8:197-203.
FTC 2016 - Future Technologies Conference 2016 6-7 December 2016 | San Francisco, United States

[7] G. Kong, D.-L. Xu, and J.-B. Yang. Clinical Decision Support Systems: A Reviewon Knowledge Representation and Inference Under Uncertainties. Int. J. Comput. Intell. Syst. 2008;1:159-67.

[8] C. Angulo, J. Cabestany, P. Rodríguez, M. Batlle, A. González, and S. de Campos. Fuzzy expert system for the detectionof episodes of poor water quality through continuous measurement. Expert Syst. Appl. 2012;39:1011-20.

[9] T. I. Liu, J. H. Singonahalli, and N. R. Iyer. Detection of Roller Bearing Defects Using Expert System and Fuzzy Logic.MechSyst Signal Process 1996;10:595-614.

[10] S. J. Russell, P. Norvig, and E. Davis. Artificial intelligence: amodern approach. Upper Saddle River, NJ: Prentice Hall; 2010.

[11] J.-B. Yang, J. Liu, J. Wang, H.-S. Sii, and H.-W. Wang. Belief rule-base inference methodology using the evidential reasoning ApproachRIMER. IEEE Trans. Syst. Man Cybern. - Part Syst Hum 2006;36:26685.

[12] G. L. Kong, D. L. Zu, and J. B. Yang. An evidence-adaptive belief rulebased clinical decision support system for clinical risk assessment in emergency care, Bonn, Germany, 2009.

[13] M. S. Hossain, K. Andersson, and S. Naznin. A Belief Rule Based Expert System to Diagnose Measles under Uncertainty. Proceedings of the 2015 International Conference on Health Informatics and Medical Systems (HIMS'15), Las Vegas, NV, USA, 2015.

[14] G. L. Kong, D. L. Zu, and J. B. Yang. An evidence-adaptive belief rulebased clinical decision support system for clinical risk assessment in emergency care, Bonn, Germany, 2009.

[15] R. Body. Clinical decision rules to enable exclusion of acute coronary syndromes in the emergency department. Doctoral Thesis. Manchester Metropolitan University, 2009.

[16] H. Skalska and V. Freylich. Web-bootstrap estimate of area under ROC curve. Aust. J. Stat. 2006;35:325-30.

[17] C. E. Metz. Basic principles of ROC analysis. Semin. Nucl. Med., 1978;8:283-98

[18] J. A. Hanley. The Robustness of the "Binormal" Assumptions Used in Fitting ROC Curves. Med. Decis. Making 1988;8:197-203. 